\title{
The Dynamics of Cavitation Bubbles
}

\author{
By M. S. PLESSET, ${ }^{1}$ PASADENA, CALIF.
}

Three regimes of liquid flow over a body are defined, namely: (a) noncavitating flow; (b) cavitating flow with a relatively small number of cavitation bubbles in the field of flow; and (c) cavitating flow with a single large cavity about the body. The assumption is made that, for the second regime of flow, the pressure coefficient in the flow field is no different from that in the noncavitating flow. On this basis, the equation of motion for the growth and collapse of a cavitation bubble containing vapor is derived and applied to experimental observations on such bubbles. The limitations of this equation of motion are pointed out, and include the effect of the finite rate of evaporation and condensation, and compressibility of vapor and liquid. A brief discussion of the role of "nuclei" in the liquid in the rate of formation of cavitation bubbles is also given.

\section{INTRODUCTION}

A

DISTINCTIVE feature of the hydrodynamics of liquids is the possibility of the coexistence of a vapor or gas phase with the liquid phase. Such two-phase flow is usually called cavitating flow, although it could as well be characterized as liquid flow with boiling. Cavitating flow has great theoretical interest in addition to the hydrodynamics involved because of the relation of this flow condition to the physical-chemical properties of the liquid. The practical significance of cavitation is of course clear. The drag of submerged bodies moving through a liquid rises when eavitation appears; similarly, the efficiency of pumps, turbines, and propellers drops with the development of cavitation; and the damage which may be produced by cavitation in these devices is well known.

The particular flow problem discussed in this paper is the flow of a liquid (water) over a submerged body which will be considered to be at rest. If $p_{0}$ denotes the static pressure, and $V_{0}$ the uniform flow velocity of the liquid at a great distance from the body, then the general character of the flow in so far as cavitation is concerned is correlated with the cavitation parameter

$$
K=\frac{p_{0}-p_{v}}{\left(\rho V_{0}^{2}\right) / 2}
$$

where $p_{v}$ is the vapor pressure of the liquid and $\rho$ its density. Obviously, one cannot expect a single constant to describe so complex a phenomenon as cavitating flow about a submerged body; however, a correlation in a qualitative way may be made with the various types of liquid flow. Three flow regimes for a given suitably shaped body will be indicated here. The first ( $K$ sufficiently large) is noncavitating flow. This state of flow consists of a liquid phase only and, with neglect of compressibility

${ }^{1}$ Associate Professor of Applied Mechanics, California Institute of Technology.

Contributed by the Applied Mechanics Division and presented at the Annual Meeting, New York, N. Y., November 28-December 3, 1948, of The American Society of Mechanical Engineers.

Discussion of this paper should be addressed to the Secretary, ASME, 29 West 39th Street, New York, N. Y., and will be accepted until October 10,1949, for publication at a later date. Discussion received after the closing date will be returned.

Note: Statements and opinions advanced in papers are to be understood as individual expressions of their authors and not those of the Society. Paper No. 48-A-107. effects, follows the same laws as are familiar in air flow. If now $K$ is made smaller, a state of flow is attained in which a relatively small number of bubbles appear near the boundary of the body. This state of flow will be taken as the second regime of flow. If $K$ is further reduced, the number of bubbles increases, until eventually they merge into one large cavity which completely encloses a portion of the body. The state of flow with a single cavity about the body is the third flow regime, and may be called cavity flow. A further reduction of $K$ brings about only an increase in the size of the cavity. These three flow conditions are illustrated in Fig. 1.

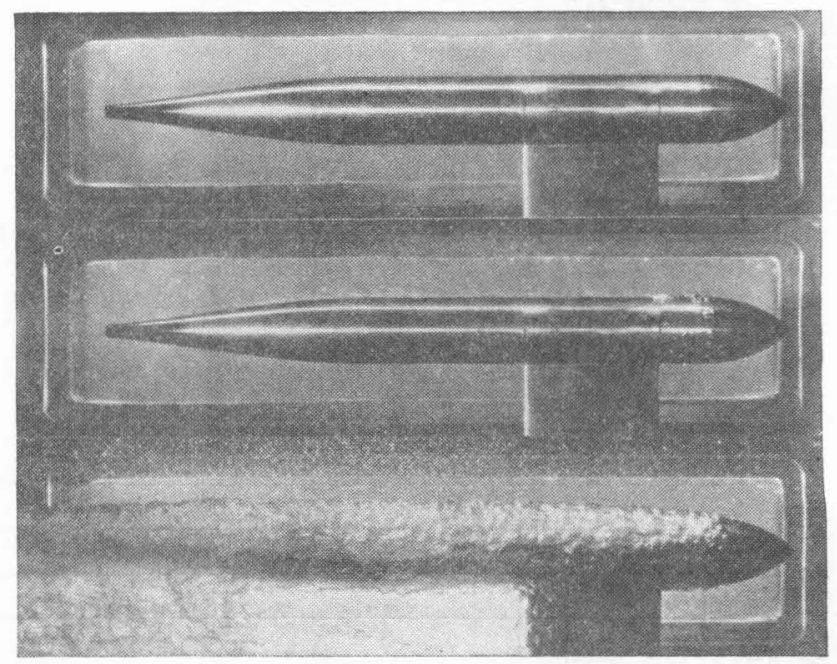

Fig. 1 Views Showing the Three Regimes of Flow (In the top view, the cavitation parameter $K=0.40$; in the center $K=$ 0.28 ; and in the bottom $K=0.18$.)

In the cavity-flow regime, the boundary of the cavity may be taken with reasonably good approximation to be a surface of constant pressure and of constant flow speed. The pressure and velocity in the flow field are fundamentally different from those in noncavitating flow. It may be remarked that, at least for two-dimensional flows, the powerful mathematical methods of the free streamline theory may be applied to the solution of cavity flow problems $(1,2) .^{2}$

The second regime of flow has here been characterized somewhat arbitrarily as the flow condition in which there is only a relatively small number of bubbles in the flow field. This limitation is made in order to get an analytic simplification. If there are only a few small bubbles, the effect of the pressure disturbance of one bubble upon another may be neglected. Further, one may suppose that the pressure field, except at the bubble, is determined in the same way as if there were no bubble cavitation. As is well known for noncavitating flow, if $p$ is the static pressure at any point in the flow field, and if $p_{0}$ and $V_{0}$ are the static pressure and flow velocity in the uniform flow at a distance from the body, then with neglect of viscous effects, the pressure coefficient

${ }_{2}$ Numbers in parentheses refer to the Bibliography at the end of the paper.

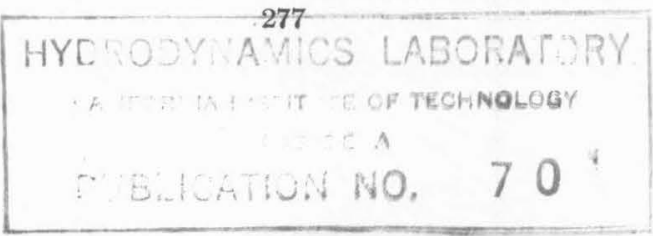




$$
C_{p}=\frac{p-p_{0}}{\left(\rho V_{0}^{2}\right) / 2}
$$

is independent of $p_{0}$ and $V_{0}$. The present assumption consists in the calculation of the static pressure $p$ in the second flow regime with the appropriate values of $p_{0}$ and $V_{0}$ from the pressure coefficient $C_{p}$ determined for noncavitating flow. This assumption that the pressure coefficient is essentially the same just before the first few cavitation bubbles appear as it is after of course is subject to experimental verification, and the necessary experiments are planned for the high-speed water tunnel in the Hydrodynamies Laboratory of the California Institute of Technology. For the present, this assumption is considered a reasonable one. It may be remarked also that as the number of bubbles increases with decreasing $K$, the pressure field should go over into that characteristic of the cavity-flow field; but, in the transition, the pressure distribution over the body should show small-scale spatial variations between the limits of the pressure field of noncavitating flow and that of the fully developed cavity flow.

\section{Experimental Observations of Cavitation Bubbles}

In the present paper an equation of motion will be developed for a cavitation bubble in a flow regime of the second type. This equation of motion will be applied to an analysis of experimental observations made in the high-speed water tunnel. Since a discussion of these experiments has been given recently by Knapp and Hollander (3), only general features will be mentioned here.

The cavitation experiments were made with a 1.5-caliber ogive for which the noncavitating pressure distribution had been measured, Fig. 2. Runs were made with tunnel velocities $V_{\mathrm{C}}$

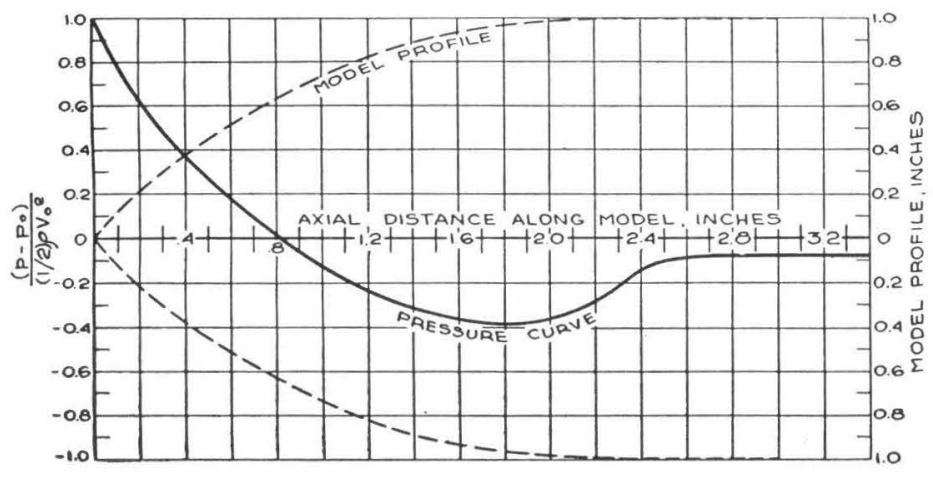

PRESSURE DISTRIBUTION ON 1.5 CALIBER OGIVE

Fig. 2 Experimentally Determined Pressure Coefficient, $C_{p}=\left(p-p_{0}\right) / 2\left(\rho V_{0}^{2}\right)$, Is Shown as a Function of AxiAL Distance Along Model

(The model profile is shown in the dotted curves with the associated seale for the profile on the right.)

from $40 \mathrm{fps}$ to $70 \mathrm{fps}$, and the static pressure $p_{0}$, was reduced until a few cavitation bubbles appeared. Photographs of these bubbles were taken on a moving film at a rate of 15,000 per sec to 20,000 per sec; a reproduction of an example of these photographs is shown in Fig. 3.

\section{Equation of Motion for a Cavitation Bubble}

Frequent reference has been made in the literature on cavitation to Rayleigh's solution for the problem of the collapse of a spherical cavity in a liquid (4). Rayleigh considered the situation in which the pressure at a distance from the bubble was constant. With this assumption, the variation of the bubble radius with time may be simply and elegantly deduced from the

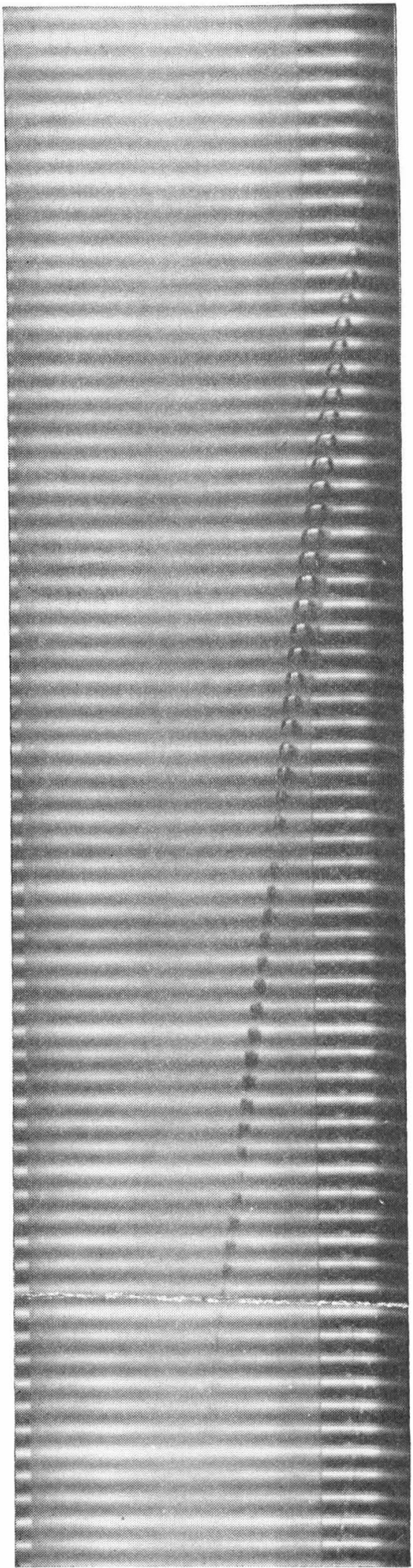

Fig. 3 This Series of Frames Shows the Bubble Danoted As BubBLE 1 
energy integral of the motion. In the present problem, the bubble moves through a region in which the pressure varies quite rapidly so that an extension of Rayleigh's theory is required. This extension may be readily carried out as follows: Consider a spherical bubble in a perfect, incompressible liquid of infinite extent, and let the origin of co-ordinates be at the bubble center which is at rest. The radius of the bubble at any time $t$ is $R$, and $r$ is the radius to any point in the liquid. Then, as is well known (5), the velocity potential for motion of the liquid with spherical symmetry is

$$
\phi=R^{2} \dot{R} / r \ldots \ldots \ldots \ldots \ldots \ldots
$$

and the Bernoulli integral of the motion is

$$
-\frac{\partial \phi}{\partial t}+\frac{1}{2}(\nabla \phi)^{2}+\frac{p(r)}{\rho}=\frac{P(t)}{\rho} \ldots
$$

where $\dot{R}=d R / d t, p(r)$ is the static pressure at $r$, and $P(t)$ is the static pressure at a distance from the bubble. Also, from Equation [3]

$$
\begin{gathered}
(\nabla \phi)^{2}=R^{4} \dot{R}^{2} / r^{4} \ldots \ldots \ldots \ldots \ldots \ldots[5] \\
\frac{\partial \phi}{\partial t}=\frac{1}{r}\left(2 R \dot{R}^{2}+R^{2} \ddot{R}\right) \ldots \ldots \ldots \ldots[6]
\end{gathered}
$$

Equation [4] will be applied at $r=R$ so that the equation of motion for the bubble radius is determined (5). One notes that

$$
\begin{gathered}
(\partial \phi / \partial t)_{r=R}=2 \dot{R}^{2}+R \ddot{R} \\
(\nabla \phi)^{2} r=R=\dot{R}^{2}
\end{gathered}
$$

so that Equation [4] becomes

$$
\frac{p(R)-P(t)}{\rho}=\frac{3}{2} \dot{R}^{2}+R \ddot{R} \ldots \ldots \ldots \ldots
$$

Equation [7] is the general equation of motion for a spherical bubble in a liquid with given external pressure $P(t)$, and with the pressure at the bubble boundary $p(R)$. One gets Rayleigh's solution as a special case with

$$
P(t)-p(R)=P_{0}(\text { a constant })
$$

and with the aid of the relation

$$
\frac{3}{2} \dot{R}^{2}+R R=\frac{1}{2 \dot{R} R^{2}} \frac{d}{d t}\left(R^{3} \dot{R}^{2}\right)
$$

Equation [7] is adapted to the present problem with the assumption that

$$
p(R)=p_{v}-2 \sigma / R .
$$

where $p_{v}$ is the vapor pressure of the water at the appropriate temperature and $\sigma$ is the surface-tension constant for water. It is thus supposed that one has to deal with the growth and collapse of a "vapor" bubble. The problem is defined when $P(t)$ is known. It will be assumed, as just discussed, that $P(t)$ is determined from the noncavitating pressure distribution over the body.

The analysis of the experimental data, and the comparison with the theory, are carried out in the following manner: The experimental data given include bubble photographs, Fig. 3, which determine the following:

1 The position of the bubble relative to the body profile as a function of time.

2 The radius $R$ of the bubble as a function of time.

Further, the tunnel temperature (and hence $p_{v}$ ) are given as well as $p_{0}$ and $V_{0}$; these data are usually combined in the specification of the cavitation parameter $K$ and the tunnel temperature. From this information, and the knowledge of the pressure distribution over the body, Fig. 2, the absolute pressure at the model surface is determined. This absolute pressure as a function of position on the model is now transformed into the function $P(t)$ from the correlation of the bubble position on the model with time. When $P(t)$ has been determined, the integration of the equation of motion (Equations [7] and [8]) may be carried out to get the radius of the bubble $R$ as a function of time. The equation of motion cannot be integrated analytically, and its integration was performed numerically. The solution is determined when two constants are specified, and these were taken to be the observed value of the maximum radius $R_{m}$ where $\dot{R}=0$. Thus the theoretical solution has been fitted to the experimental curve only at the peak of the radius-time curve. The theoretical curve was then determined by integrating forward (the collapse portion) and backward (the growth portion) from this one point. A comparison of the calculations with the measured values is shown in Figs. 4, 5, 6, and 7. The agreement is considered satisfactory, particularly since it must be emphasized that precise experimental data are difficult to obtain. The theoretical radius-time curve is quite sensitive to the $P(t)$ function; for the experiments thus far analyzed, it is believed by the experimental workers that the cavitation parameter $K$ has not been determined with quite the necessary accuracy. Further, there are some difficulties in the determination of the bubble outlines with precision. That this is the case is not surprising since one is requiring considerable photographic detail throughout a process which lasts for a time of the order of a millisecond. It must also be pointed out that there are approximations involved in applying the theoretical equation to the experimental situation. These approximations will now be considered.

\section{Theoretical Approximations}

The Pressure Field. It has been supposed that the pressure field, $P(t)$, acting on the bubble is determined from the pressure distribution over the model. It is clear that, in the initial stages of cavitation of present interest, the bubbles will form as close to the model surface as possible since the pressures take their lowest values there. However, it also has been assumed that the bubble is acted on by a spherically symmetric field. Since the bubble is of finite extent and since the pressure field has definite pressure gradients both along the model and normal to it, it is clear that a simplification has been introduced. These pressure gradients would be a source of asymmetry in bubble shape, and there is some evidence of this asymmetry. It is believed that the approximation made is not such as to obscure the essential details of the growth and collapse; space gradients in the pressure field are here regarded as a second-order effect.

It also has been assumed that the bubble is in a liquid of infinite extent, and it is evident that the bubble grows and collapses in the neighborhood of the model surface. This asymmetry in the fluid field has an effect which may be pointed out as follows: As compared with the experimental situation, the theory would exaggerate the importance of the liquid inertia (this inertia leads to the term in $\dot{R}^{2}$ in Equation [7]). Comparison of the theoretical curve with the experimental points would seem to indicate some overestimate of this inertia term where $R$ is small, i.e., near the beginning of the growth and toward the end of the collapse.

The presence of the model surface has an additional effect on the flow field in its neighborhood which arises from the boundary layer. The thickness of this boundary layer may be estimated from the Blasius formula, and for the present flow conditions 


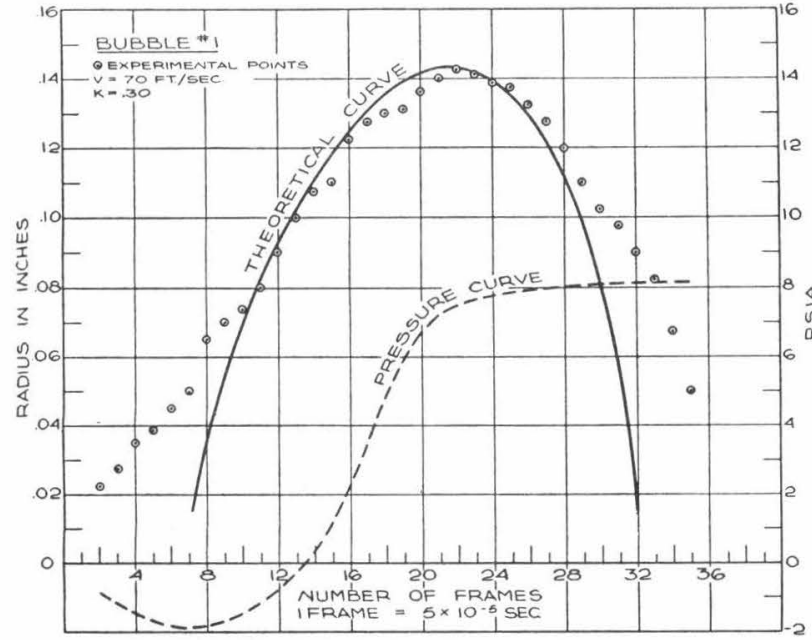

FIG. 4

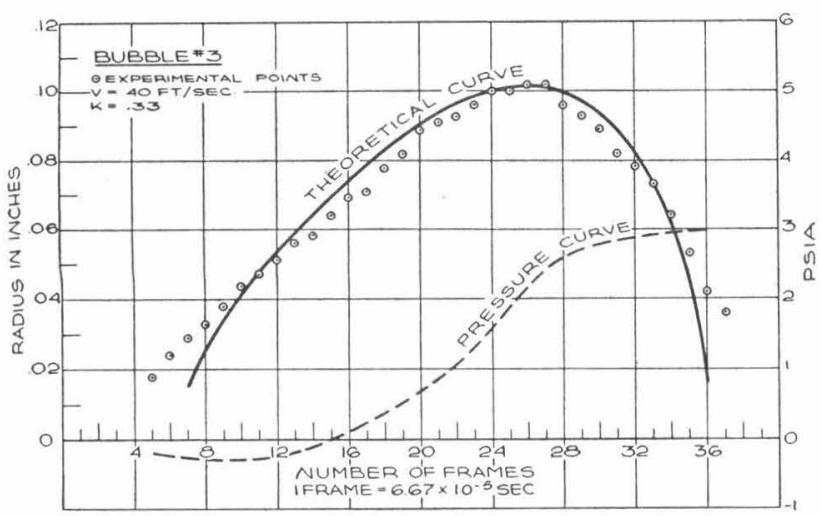

FIG. 6

leads to a thickness of the order of $6 \times 10^{-3}$ in. On the basis of this estimate, the effect of the boundary layer will be neglected. It should be noted that the present measurements extend to minimum bubble sizes larger than this boundary-layer thickness although some reduction in the effective value of $\dot{R}$ should be expected for very small $R$.

An experimental source of apparent asymmetry in bubble shape might be supposed to arise from an overestimate of the bubble dimension in the direction of its motion which would be produced by its motion during the time of light exposure $(1.5 \times$ $10^{-6} \mathrm{sec}$ ). However, this blurring would give an apparent extension of the image by approximately $10^{-3}$ in. so that this error is not particularly significant.

Temperature and Pressure Conditions in Bubble. It has been assumed in the theoretical calculations that the vapor pressure, $p_{v}$, in the bubble, and hence the bubble temperature, remain constant. Clearly, heat must be applied to the bubble to evaporate water and maintain the vapor pressure during growth, and heat of condensation must be removed during collapse. The temperature changes required may be estimated readily. Consider a bubble with maximum radius $R_{m}$ which has a growth time $\tau$. The total mass of vapor which is evaporated into the bubble is $(4 \pi / 3) R_{m}{ }^{3} \rho^{\prime}$, where $\rho^{\prime}$ is the vapor density. The total heat required is

$$
Q=(4 \pi / 3) R_{m}^{3} \rho^{\prime} L
$$

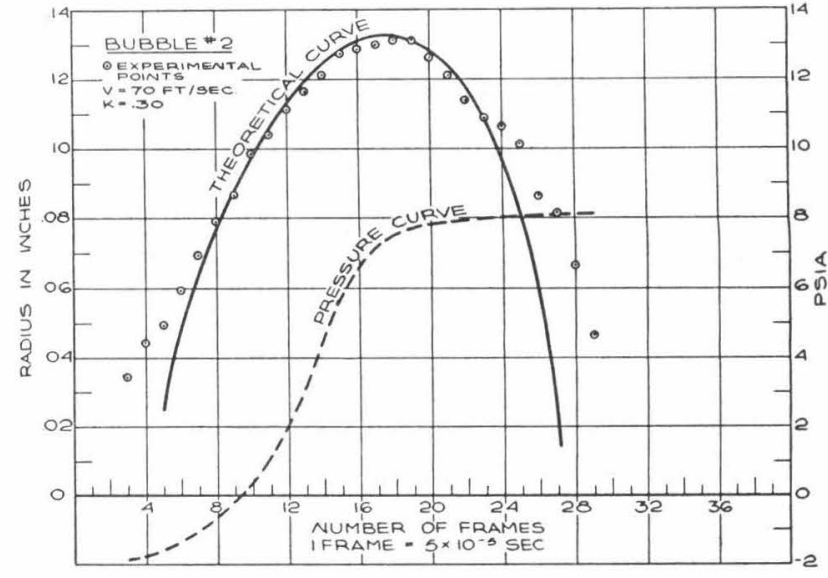

FIG. 5

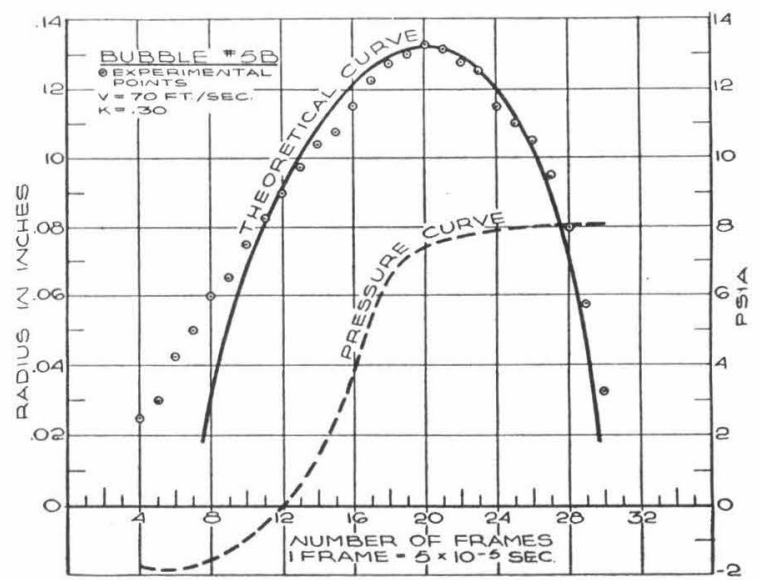

FIg. 7

where $L$ is the latent heat of evaporation. Thus for a bubble which grows to a maximum radius $R_{m}=0.10 \mathrm{in}$. in 20 frames $\left(\tau=10^{-3} \mathrm{sec}\right)$, the mass of vapor is $1.17 \times 10^{-6} \mathrm{grams}$, and $Q=$ $6.8 \times 10^{-4}$ calories. This heat is taken out of a water layer surrounding the bubble. If the thermal diffusivity of water is $D$ $\left(D=1.43 \times 10^{-3} \mathrm{sq} \mathrm{cm}\right.$ per sec), then the order of magnitude of the thickness $d$ of the water layer from which this heat is conducted is

$$
d \approx \sqrt{D \tau}
$$

and for $\tau=10^{-3} \mathrm{sec}, d \approx 1.2 \times 10^{-3} \mathrm{~cm}$. The volume of the water layer from which this heat comes is of the order of magnitude $4 \pi R_{m}{ }^{2} d$, and, in the present example, the corresponding mass of water is $1.0 \times 10^{-3} \mathrm{~g}$. Finally, the temperature drop of this water layer is

$$
\begin{aligned}
\Delta T & \approx \frac{(4 \pi / 3) R_{m}{ }^{3} \rho^{\prime} L}{4 \pi R_{m}^{2} d \rho c}=\frac{R_{m}}{3 d} \frac{\rho^{\prime} L}{\rho c} \\
& \approx \frac{R_{m}}{3 \sqrt{D \tau}} \frac{\rho^{\prime} L}{\rho c}
\end{aligned}
$$

where $c$ is the specific heat of water. In the present example, $\Delta T$ (growth) $\approx 0.7 \mathrm{deg} \mathrm{C}=1.3 \mathrm{deg} \mathrm{F}$. A typical value of collapse time is $\tau \approx 10$ frames $=0.5 \times 10^{-3} \mathrm{sec}$, and the corresponding 
temperature change, estimated in this same way, is

$$
\Delta T \text { (collapse) } \approx 1 \operatorname{deg} \mathrm{C}=1.8 \mathrm{deg} \mathrm{F}
$$

It is apparent that these temperature changes are insignificant so that one may take the bubble boundary to have a constant temperature, essentially the same as the water temperature, and a constant value of $p_{v}$.

This conclusion cannot be accepted unconditionally, however, since evaporation, or condensation, is a process which takes place at a finite rate and, if this rate is not sufficiently high to keep up with the rate of volume change of the bubble, the vapor in the bubble will behave more like a permanent than a condensable gas. This effect definitely limits the range of validity of the particular assumption, $p_{v}=$ const, toward the end of the collapse phase where the radial velocity $\dot{R}$ increases rapidly. This trend toward rapid increase in the calculated radial velocity is illustrated in Fig. 8 . The rate of evaporation,

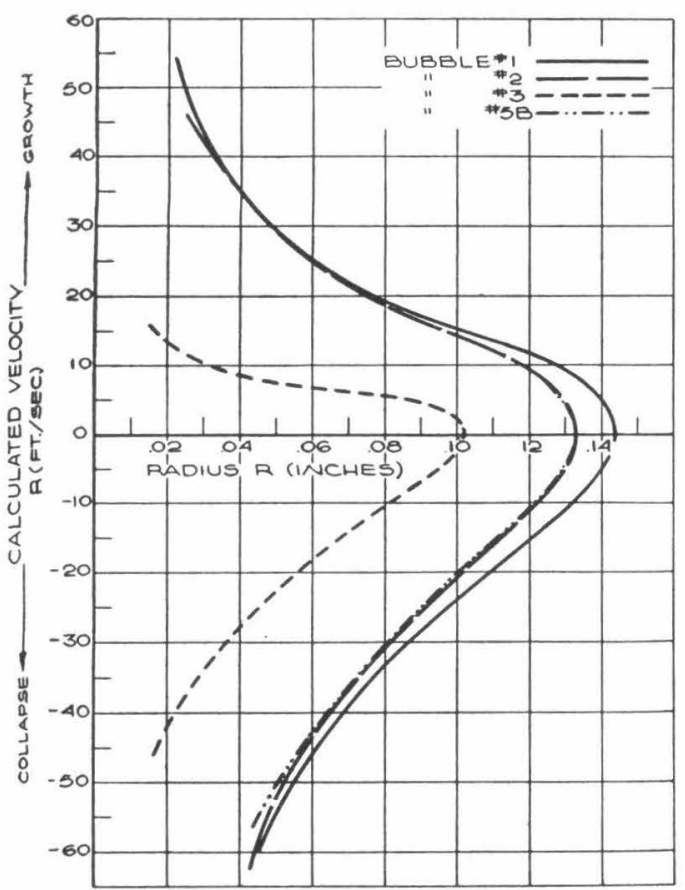

Fig. 8 Calculated Radial Velocities $\dot{R}$, Are Shown as a FuncTION OF BuBbLE Radius $R$

or condensation, can be estimated from elementary kinetic theory which says that the mass of gas evaporated (or condensed) per unit area per unit time at an absolute temperature $T$ is

$$
j=p_{v} \sqrt{\frac{M}{2 \pi B T}} \ldots \ldots \ldots \ldots \ldots \ldots[9]
$$

where $p_{v}$ again denotes the vapor pressure for a vapor with molar mass $M$, and $B$ is the gas constant. If one assumes that the vapor obeys the perfect, gas law

$$
p_{v}=\frac{\rho^{\prime}}{M} B T
$$

which is reasonably accurate in the temperature range of interest (6), Equation [9] may be written

$$
j=\rho^{\prime} \sqrt{\frac{B T}{2 \pi M}}=\rho^{\prime} V .
$$

where $V=\sqrt{B T / 2 \pi M}$ is the desired velocity to be associated with the rate of the evaporation or condensation process. For the present problem, at $22.2 \mathrm{C}=72 \mathrm{~F}, V$ is approximately 150 mps $\approx 500 \mathrm{fps}$. Hence unless $\dot{R}$ is appreciably less than this value, one may not assume the constant value for $p_{v}$. During the collapse, when $\dot{R}$ approaches or exceeds this value, the collapse velocity would tend to be decreased because the vapor will begin to show a rising pressure as it behaves like a permanent gas.

A further effect of interest is the shock loss which will appear in the vapor when $\dot{R}$ reaches the gas acoustic velocity. The effects of compressibility both in the vapor and in the liquid will not be considered here, although the problems posed by them are of great interest. A solution of these problems will be decisive for the quantitative determination of the high pressures arising toward the end of the bubble collapse, the regrowth or subsequent oscillations of the bubble, and the sound energy radiated.

Air Content in Bubble and Role of Nuclei in Formation of Bubbles. The assumption has been made that any air contained in the bubble does not affect the dynamics of the bubble growth and collapse over the range of bubble sizes which have been measured and analyzed here. This assumption might be considered questionable since the water-tunnel flow experiments are made with water containing an appreciable concentration of dissolved air. Furthermore in the region of flow in which the bubble behavior is studied, the liquid pressure is considerably below the liquid static pressure $p_{0}$ at a distance from the model. Hence one should expect that the water is supersaturated with dissolved air and that diffusion of air into the bubble would take place.

An analytic solution for such a diffusion problem has been carried out by P. S. Epstein and the author, the details of which will be presented elsewhere. For the present discussion it is necessary only to say that the diffusion process is so slow that it does not contribute appreciably to any alteration in the air content of the bubble.

As will be pointed out later, the initial air content of a bubble is very small so that over the range of bubble sizes which are observed and to which the present calculations have been applied, the effect of the air may be neglected. It must be emphasized, however, that the small mass of air in the bubble plays a most important role in the initial stages of bubble growth, and also may enter in the final stages of the bubble collapse. The initial stages of bubble growth in which the air content would be of significance, refer to bubble dimensions which are beyond the present range of experimental observation. Similarly, the final stages of bubble collapse in which the compressibility of air, water vapor, and liquid are of importance, refer to bubble dimensions which lie within the last frame photographed. ${ }^{3}$

A few remarks, nevertheless, may be made concerning the initial formation of the bubble. It is the present view that the formation of a bubble in cavitating flow, or in boiling, begins from a nucleus within the liquid containing air, or vapor, or both. Such gas-phase nuclei are ordinarily submicroscopic in size, and become evident only upon growth of the nuclei through pressure reduction in the liquid (reduction in the function denoted previously by $P[t]$ ), or through elevation of temperature (increase in the function denoted by $p[R])$. The absence of such nuclei means that the very large forces of surface tension must be overcome to initiate cavitation or boiling. It is well known that degassed pure liquids can withstand very large tensions,

${ }^{3}$ Knapp and Hollander (3) assumed that, over the present range of observation, the bubble contains essentially only water vapor. The present discussion supports this view. 
or may be superheated considerably, without the formation of cavities and bubbles.

Recently, Harvey (7) and subsequently Pease and Blinks (8) have shown experimentally that water saturated with air also has high tensile strength, provided it is denucleated. Harvey's method of denucleation of water saturated with air consists in putting the solution under high pressures (of the order of 10,000 psi) for several minutes. The air nuclei are squeezed into solution so that when the solution is brought back to atmospheric pressure it does not cavitate under the tensions which freely produced cavitation before the pressurization. These same pressure-treated air-water solutions also can be superheated by as much as 60 to 80 deg $\mathrm{C}$ without boiling.

Presumably in ordinary untreated water the nuclei which contain gas and vapor are stabilized on small solid particles. The presence of a solid, or third phase, is indicated since the surface energy of a bubble bounded by a solid surface and a liquid surface may be very low. Methods whereby the probable rate of formation of nuclei as determined by the surface energy may be calculated have been discussed by Becker and Döring (9) and Kaischew and Stranski (10). The aim of the theory is to calculate the tensile strength of liquids, but it should be applicable also to the statistics of the number of nuclei which should grow to macroscopic bubbles for given conditions of liquid temperature and pressure.

\section{Conclusion}

The main purpose of the present discussion, aside from touching upon problems which still await quantitative solution, has been to point out the following: Liquid flow can be divided into the three regimes mentioned; and, since the noncavitating regime and the single-cavity regime may be considered to be on a quantitative basis, the main concern here has been a clarification of the second, or bubble, regime of flow. Also, it has been remarked that an interesting experiment would be the measurement of the pressure distribution over a body in this second regime of flow. It has been shown that the macroscopic behavior of cavitation bubbles may be explained reasonably well by a fairly simple equation.

Finally, it may be pointed out that the macroscopic behavior of the bubbles formed in a boiling liquid may be considered as entirely analogous to the cavitation bubbles more specifically considered here. The growth of bubbles in a liquid has great interest at present in the problem of increasing the heat transfer from a heated solid to a liquid.

\section{ACKNOWLEDGMENTS}

The study was carried on in the Hydrodynamics Laboratory of the California Institute of Technology. It forms a part of the activities of Contract NOrd-9612 which is jointly supported by the Research and Development Division of the Bureau of Ordnance and the Fluid Mechanics Branch of the Office of Naval Research.

The author wishes also to acknowledge the assistance of Mr. F. H. Brady and Mr. J. M. Green in the numerical computations.

\section{BIBLIOGRAPHY}

1 "Hydrodynamies," by H. Lamb, Dover Publications, New York, N. Y., sixth edition, 1945, chapt. 4, pp. 73-78.

2 "Theoretical Hydrodynamies," by L. M. Milne-Thompson, Macmillan \& Company, London, England, 1938, chapt. 13.

3 "Laboratory Investigations of the Mechanism of Cavitation," by R. T. Knapp and A. Hollander, Trans. ASME, vol. 70, 1948, p. 419 .

4 "Pressure Developed in a Liquid During the Collapse of a Spherical Cavity," by Lord Rayleigh, Philosophical Magazine, vol. 34, 1917, pp. 94-98 (Collected Papers, vol. 6, pp. 504-507).

5 "Hydrodynamics," by H. Lamb, Dover Publications, New York, N. Y., sixth edition, 1945, chapt. 5, art. 91a.

6 "Properties of Ordinary Water-Substance," by N. E. Dorsey, Reinhold Publishing Corporation, New York, N. Y., 1940, table 250, pp. 575-576.

7 "On Cavity Formation in Water," by E. Newton Harvey, W. D. McElroy, and A. H. Whiteley, Journal of Applied Physics, vol. 18,1947 , p. 162 .

8 "Cavitation From Solid Surfaces in the Absence of Gas Nuclei," by D. C. Pease and L. R. Blinks, Journal of Physical and Colloid Chemistry, vol. 51, 1947, p. 556.

9 "Kinetische Behandlung der Keimbildung in Übersättigen Dämpfen," by R. Becker and W. Döring, Annalen der Physik, vol. 24 , series 5 , p. 719 .

10 "Zur Kinetischen Ableitung der Keimbildungsgeschwindigkeit," by R. Kaischew and I. N. Stranski, Zeitschrift für Physikalische Chemie, (B), vol. 26, 1934, p. 317. 